\title{
Emergency aortic valve replacement complicated by unsuspected pheochromocytoma
}

\author{
Rihito Tamaki ${ }^{1}$, Manabu Yamasaki ${ }^{1}$, Hiroyuki Nishi ${ }^{2}$, Kunihiko Yoshino ${ }^{1}$, Kohei Abe ${ }^{1}$, and \\ Hiroyasu Misumi ${ }^{1}$ \\ ${ }^{1}$ St Luke's International University \\ ${ }^{2}$ Osaka General Medical Center
}

January 6, 2021

\begin{abstract}
A 53-year-old male undergoing emergency aortic valve replacement for infective endocarditis developed a hypertensive crisis early during the operation. Suspecting a pheochromocytoma, intravenous phentolamine was immediately administered, after which the procedure was completed as scheduled. Although quite rare, a pheochromocytoma can be encountered during emergency open heart surgery, thus early recognition of abnormal blood pressure change and appropriate management are important. Here, we present details of blood pressure control mainly by phentolamine use in this case to demonstrate effective management of a hypertensive crisis during emergency cardiac surgery because of a pheochromocytoma.
\end{abstract}

\section{INTRODUCTION}

A pheochromocytoma is a rare tumor that can cause serious complications during surgery, with $25-50 \%$ of hospital deaths in patients with unmanaged or unknown pheochromocytoma occurring during anesthesia induction or other operative procedures. ${ }^{1}$ Herein, we report emergency aortic valve replacement (AVR) for infectious endocarditis (IE) complicated by a hypertensive crisis due to unsuspected pheochromocytoma.

\section{CASE REPORT}

A 53-year-old male was admitted after occurrence of dyspnea for two weeks and repeated transient cerebral ischemic attacks of right hemiplegia occurred over a 4-day period. The patient was extremely obese, with body weight of $130 \mathrm{~kg}$ and BMI of 44.2. He was prescribed immunosuppressive medication for pyoderma gangrenosum and had type 2 diabetes mellitus, hypertension, sleep apnea syndrome, and history of ablation. Blood pressure (BP) was 133/92 $\mathrm{mm} \mathrm{Hg}$, pulse was 119/minute, and respiratory rate was $30 /$ minute with an $\mathrm{O} 2$ saturation of $94 \%$ via nasal cannula at $3 \mathrm{~L} /$ minute. Chest radiography findings showed pulmonary congestion. Transthoracic echocardiography revealed a $2.7 \times 9-\mathrm{mm}$ mobile mass on the noncoronary cusp of the aortic valve, which initiated IE. Based on mobile mass with neurological dysfunction and heart failure progression, there was no time to wait and an emergency AVR was performed.

Upon admission to the operating room, BP was $200 / 103 \mathrm{mmHg}$, then rose during endotracheal suction to $342 / 167 \mathrm{mmHg}$ in association with sinus tachycardia at 105/minute (Figure 1). Initially, improper medication administration was suspected and all medications were replaced, though hypertensive crisis recovery was not obtained. We decided to continue the operation because of heart failure progression and neurological complications. However, even with administration of routine anti-hypertensive drugs (nitroglycerin, nicardipine), a steady hemodynamic condition was difficult to maintain due to repeated BP elevation and the hypertensive crisis continued after initiation of cardiopulmonary bypass (CPB). It was then noted that a retroperitoneal mass had been seen at a previous hospital examination, which led us to suspect a pheochromocytoma (Figure 
2A) and an intermittent intravenous infusion of phentolamine was started. After finishing the AVR procedure, $\mathrm{CPB}$ was weaned with intravenous phentolamine and phenylephrine for stabilization of $\mathrm{BP}$, with a continuous infusion of both phentolamine and norepinephrine needed at the end of the operation. Vegetation was noted on the left-ventricular side of the noncoronary cusp. A 25-mm mechanical valve (Masters 25-mm prosthesis, Abbott St. Jude Medical, St. Paul, USA) was implanted in an intra-annular position.

In the early postoperative period, noradrenaline combined with phentolamine was still required, due to intermittent hypotension, then after discharge from the intensive care unit antihypertensive medication was stopped due to continued orthostatic hypotension symptoms. A close examination for possible pheochromocytoma was performed one month after the operation by an endocrinologist, which showed urinary metanephrine plus normetanephrine at $4.2 \mathrm{mg}$ /day (reference $0.15-0.41 \mathrm{mg} /$ day) and free noradrenaline at $343 \mu \mathrm{g} /$ day $(31-160 \mu \mathrm{g} /$ day $)$. Additionally, ${ }^{123}$ I-metaiodobenzylguanidine scintigraphy revealed abnormal accumulation in the left adrenal gland (Figure 2B) and a diagnosis of pheochromocytoma was confirmed. After six weeks of antimicrobial treatment, the patient was transferred to a rehabilitation hospital (postoperative day 56) and scheduled for adrenalectomy after recovery of general condition.

\section{COMMENT}

A pheochromocytoma is a catecholamine-secreting tumor that arises from chromaffin cells of the adrenal medulla. Patients with unrecognized pheochromocytoma who undergo anesthesia for unrelated surgery have significantly high risk for a hypertensive crisis. Five previous reports of cardiac surgeries completed despite complications encountered due to unsuspected pheochromocytoma are known, including coronary artery bypass grafting (CABG), ${ }^{2} \mathrm{AVR},{ }^{3}$ concurrent AVR and $\mathrm{CABG},{ }^{4}$ mitral valve replacement, ${ }^{5}$ and even a heart transplant operation, ${ }^{6}$ each an elective procedure. As noted in a study of cases of aortic dissection complicated by unsuspected pheochromocytoma, ${ }^{7}$ reports of emergency cardiac surgery for patients with unsuspected pheochromocytoma are limited. Generally, while the incidence of pheochromocytoma is infrequent, it can be encountered during various emergency as well as elective cardiac procedures. Identification of such a situation is an unsolved issue, though a systematic diagnosis and treatment strategy is necessary, because surgeons sometimes must continue the operation for various reasons, such as heart failure or neurological complications. Our case provides valuable detailed information for establishment of appropriate management of a hypertensive crisis in a case of unsuspected pheochromocytoma.

Cardiac surgery requires precise control of hemodynamics to permit bypass, and myocardial and organ protection procedures, as well as return to a normal loaded heart as circulatory support is withdrawn. Therefore, appropriate BP control is important when a hypertensive crisis emerges during cardiac surgery. For precise management of $\mathrm{BP}$ in patients with a pheochromocytoma, a combined alpha- and beta-adrenergic blockade is the most commonly implemented strategy. In a previous case with a pheochromocytoma suspected during the operation, an alpha-adrenergic blockade such as phentolamine and beta-adrenergic blockade such as propranolol were used in combination with nitroprusside for BP management. ${ }^{5}$ Our patient suffered from an unpredicted hypertensive crisis that occurred after induction of anesthesia. Before we suspected a pheochromocytoma, nicardipine and nitroglycerin were used, then when the condition was revealed, those were promptly replaced by phentolamine, which was effective for lowering BP. While there were various differential diagnoses for the cause of the hypertensive crisis, including neurological injury, preoperative CT imaging helped to confirm the diagnosis of pheochromocytoma in this case, which resulted in adequate BP management using a combined alpha-adrenergic blockade. Refractory or persistent hypertension may sometimes be associated with a serious disease in cases of cardiac surgery, thus it is important to consider a differential diagnosis, including pheochromocytoma, with early intervention needed to overcome this critical situation.

During cardiovascular surgery, once CPB is established, it is expected that hemodynamics will stabilize due to hemodilution. However, Brown et al. reported that CPB itself may contribute to the severity of hypertension, while exposure to cold can increase catecholamine secretion from a pheochromocytoma. ${ }^{8}$ In this case, it was difficult to control BP even after starting CPB. Careful hemodynamic management of a hypertensive crisis caused by a pheochromocytoma is necessary even after CPB is established. It is also 
important to note that vasopressor use was needed to maintain adequate BP during and after our operation, though that can potentially make management difficult. To overcome such complications, findings in our

case provide useful detailed information of this rare situation from a pharmacological management point of view.

\section{Data Availability Statement}

Data sharing is not applicable to this article as no new data were created or analyzed in this study.

\section{References}

1. Sutton MG, Sheps SG, Lie JT. Prevalence of clinically unsuspected pheochromocytoma. Review of a 50-year autopsy series. Mayo Clin Proc 1981;56:354-360.

2. Walker T, Bail DHL, Schmid E, Haeberle L, Kuehne H, Ziemer G. Challenging the advanced: Cardiac surgery without awareness of a pheochromocytoma. Thorac Cardiovasc Surg 2006;54:498-499

3. Singh AK, Nguyen PN. Refractory ventricular tachycardia following aortic valve replacement complicated by unsuspected pheochromocytoma. Thorac Cardiovasc Surg 1993;41:372-373.

4. Soon JL, Gibson FAM, Janas S, Pillai R. Advantages of sutureless aortic valve in a patient with unsuspected pheochromocytoma. Innov Technol Tech Cardiothorac Vasc Surg 2010;5:306-308.

5. Robertson JM, Kozyra-Cushen C, Stead SW, Lee J, Mulder DG. Mitral valve replacement complicated by unsuspected pheochromocytoma. J Thorac Cardiovasc Surg 1986;91:630-633.

6. Dalby MCD, Burke M, Radley-Smith R, Banner NR. Pheochromocytoma presenting after cardiac transplantation for dilated cardiomyopathy. J Hear Lung Transplant 2001;20:773-775.

7. Runyan B, Hanak CR, Mahendiran S, Allamaneni S, Vester S. Type A Aortic Dissection Complicated by Pheochromocytoma. Ann Thorac Surg 2019;107:e13-14.

8. Brown P, Caplan RA. Recognition of an unsuspected phaeochromocytoma during elective coronary artery bypass surgery. Can Anaesth Soc J 1986;33:785-789

\section{Acknowledgement}

We thank EBM skill up project by Young Kwang Park, PhD and Hikaru Matsuda, MD.

\section{Conflict of Interest}

The authors declare that there is no conflict of interest.

\section{Legends}

Figure 1.

Severe changes in blood pressure, and vasodilator and vasopressor administration sequence.

$\mathrm{HR}=$ heart rate, $\mathrm{mBP}=$ mean blood pressure, $\mathrm{sBP}=$ systolic blood pressure

Figure 2 .

A: Left-side retroperitoneal mass noted at previous hospital (yellow arrow). B: Intense uptake of ${ }^{123} \mathrm{I}-$ metaiodobenzylguanidine by left adrenal shown by postoperative scintigraphy. 

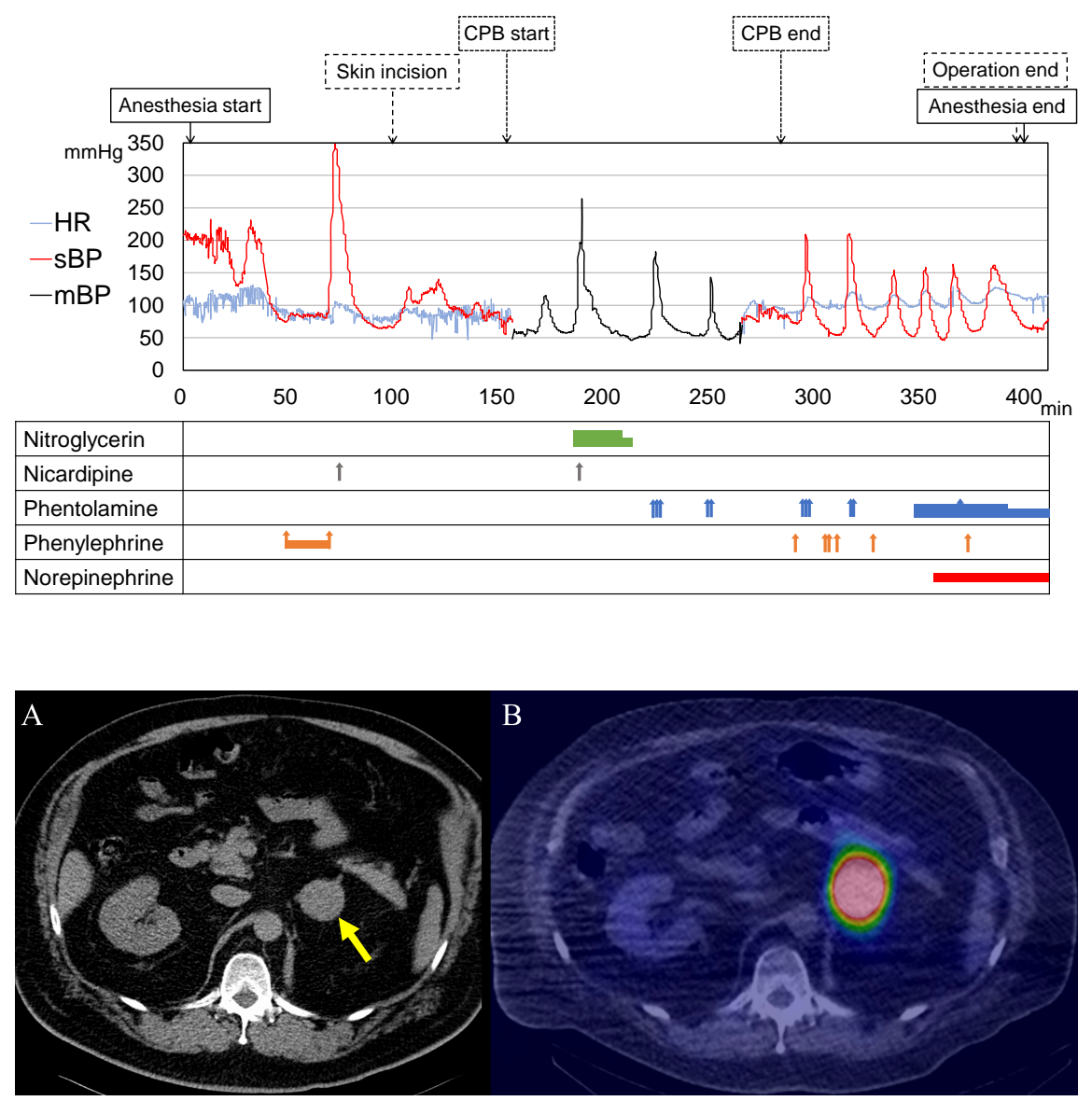